\title{
¿Influyen las aves migratorias neárticas en el patrón estacional de aves de los humedales costeros?
}

\author{
Do influence Nearctic migratory birds in the seasonal pattern of coastal wetlands birds?
}

\author{
Álvaro García-Olaechea ${ }^{1,2}$, César Chávez-Villavicencio ${ }^{1,3 *}$, Elier Tabilo-Valdivieso ${ }^{1,3}$ \\ 1 Centro Neotropical de Entrenamiento en Humedales \\ 2 Centro de Investigación Biodiversidad Sostenible - BioS \\ 3 Programa de Doctorado en Biología y Ecología Aplicada. Universidad Católica del Norte - Universidad La Serena. Campus Guayacán. Calle Larrondo \\ N¹281, Coquimbo - Chile \\ *Autor para correspondencia \\ Email Álvaro García-Olaechea: agarcia@centroneotropical.org \\ Email César Chávez-Villavicencio: cchavez@ucn.cl \\ Email Elier Tabilo-Valdivieso: etabilo@centroneotropical.org
}

\section{Resumen}

Si la llegada en primavera-verano de las aves limícolas migratorias neárticas influye en el incremento de la riqueza y abundancia de los ensambles de aves en los humedales costeros entonces, tanto la riqueza como la abundancia de este grupo de aves disminuirán hacia el otoño. Para comprobarlo se usó como modelo de estudio al Manglar San Pedro de Vice donde se estableció que la disminución de la riqueza de aves en otoño podría deberse al retorno de las especies migratorias neárticas hacia el hemisferio norte. Por tanto, el objetivo fue determinar la disminución de la riqueza y abundancia de aves limícolas migratorias neárticas. Se realizaron 10 muestreos quincenales entre diciembre del 2010 y abril del 2011, recorriendo una distancia de $4.5 \mathrm{~km}$ desde el norte del canal de marea preferentemente cuando la marea estaba bajando. La disminución de la riqueza se analizó con una regresión de Poisson, la disminución de la abundancia se hizo a través de los promedios de abundancias con un modelo lineal generalizado. Se registraron 19 especies de aves limícolas consideradas como migratorias neárticas. La riqueza de especies y la abundancia mostraron una disminución estadísticamente significativa de acuerdo a los intervalos de confianza de las pendientes respectivas. Se concluye que las aves limícolas migratorias neárticas sí influyen en los patrones estacionales de los ensambles de aves en los humedales costeros en Perú.

Palabras clave: Aves Limícolas; Costa Pacífica; Diversidad; Ensamble Aves; Manglares.

\section{Abstract}

If the spring-summer arrival of migratory Nearctic waders influences the increase in richness and abundance of bird assemblages in coastal wetlands then both the richness and abundance of this group of birds will decline by the fall. To verify this, the San Pedro de Vice mangrove was used as a model of study where it was established that the fall in bird species in autumn could be due to the return of migratory species to the northern hemisphere. Therefore, the objective was to determine the decrease in richness and abundance of migratory Nearctic waders. Ten biweekly surveys were conducted between December 2010 and April 2011, covering a distance of $4.5 \mathrm{~km}$ from the north of the tidal channel, preferably when the tide was descending. The decrease in richness was analyzed with a Poisson regression, the decrease in abundance was done through the abundance mean with a generalized linear model. There were 19 species of waders considered as migratory Nearctic. Species richness and abundance showed a statistically significant decrease according to the confidence intervals of the respective slopes. It is concluded that migratory Nearctic wader influence the seasonal patterns of bird assemblages in coastal wetlands in Peru.

Keywords: Bird Assembly; Diversity; Mangroves; Pacific Coast; Wader.

Citación:

García-Olaechea A., C. Chávez-Villavicencio, E. Tabilo-Valdivieso. 2018. ¿Influyen las aves migratorias neárticas en el patrón estacional de aves de los humedales costeros? Revista peruana de biología 25(2): 117 - 122 (Mayo 2018). doi: http://dx.doi.org/10.15381/rpb.v25i2.13281

$$
\begin{array}{ll}
\text { Presentado: } & 10 / 06 / 2017 \\
\text { Aceptado: } & 26 / 02 / 2018
\end{array}
$$

Publicado online: $28 / 05 / 2018$
Fuentes de financiamiento: Servicio Forestal de los Estados Unidos (USFS); CRIMBI (Copper River International Migratory Bird Initiative).

Información sobre los autores:

AG-O, CC-V, ET-V: Realizaron el diseño de muestreo. AG-O: Tomó datos en campo. AG-O, CC-V , ET-V: Realizaron el análisis estadístico de los datos, redactaron, revisaron y aprobaron el manuscrito.

Los autores no incurren en conflictos de intereses. 


\section{Introducción}

En la región desértica de la costa del Perú, los humedales son ecosistemas claves para el mantenimiento de la vida silvestre (Tabilo et al. 2016). Para las aves, residentes o migratorias, ofrecen un hábitat óptimo para desarrollarse tanto temporalmente como a lo largo de su vida, para anidar o alimentarse (Bildstein et al. 1991, Kushlan 1993, Pulido 2003, Gauthier et al. 2005). El conocimiento sobre el uso temporal de los humedales por algunas especies de aves se debe a estudios de la variación estacional en diferentes humedales costeros (Pulido 1987, Hughes 1991, Keddy 2002, Chávez-Villavicencio 2005, Torres et al. 2006, Álvarez 2007, Vizcarra 2008, Gonzáles et al. 2011). Los autores citados concluyen que la riqueza y abundancia de aves en los humedales es mayor en los meses de verano-otoño (diciembre-junio), con respecto a los meses de invierno-primavera (julio-noviembre), debido a la llegada y partida de las especies de aves migratorias, respectivamente.

Entre las aves migratorias que arriban a los humedales se encuentra el grupo de las neárticas, es decir, las que pasan su época reproductiva al norte del trópico de cáncer y el resto de su vida al sur del mismo (Rappole et al. 1993). Dentro de las aves migratorias neárticas se encuentran las aves limícolas conocidas comúnmente como chorlos, playeros o aves de orilla. Estas aves se alimentan en zonas limosas y a orillas de mar. Taxonómicamente pertenecen al Orden Charadriiforme, siendo las familias Charadriidae, Haematopodidae, Recurvirostridae y Scolopacidae las que presentan especies netamente limícolas (Koepcke 1964, Canevari et al. 2001).
Por tanto se considera que, si la llegada en primavera-verano de las aves limícolas migratorias neárticas influye en el incremento de la riqueza y abundancia de los ensambles de aves en los humedales costeros entonces, tanto la riqueza como la abundancia de este particular grupo de aves disminuirán hacia el otoño, época en que comienza el retorno migratorio hacia el norte.

Para comprobar la disminución de la riqueza y abundancia en el otońo de los ensambles de aves en humedales costeros a causa de la partida aves limícolas, se usó como modelo de estudio al Manglar San Pedro de Vice. Este manglar es un humedal de importancia mundial (The Ramsar Convention 2010), miembro de la Red Hemisférica de Reservas de Aves Playeras (RHRAP 2010) y un Área Importante para la Conservación de las Aves IBA 012- (Angulo 2009), donde Chávez-Villavicencio (2005) estableció que la disminución de la riqueza de aves en otoño podría deberse al retorno de las especies migratorias neárticas hacia el hemisferio norte (incluyendo las limícolas). Por tanto, el objetivo de este estudio fue determinar la disminución de la riqueza y abundancia de aves limícolas migratorias neárticas en el Manglar San Pedro de Vice.

\section{Materiales y métodos}

Área de estudio.- El Manglar de San Pedro de Vice se ubica dentro de la ecorregión desierto de Sechura (5²9' - 5³3'S y $\left.80^{\circ} 52^{\prime}-80^{\circ} 54^{\prime} \mathrm{W}\right)$, a $50 \mathrm{~km}$ de la ciudad de Piura y $20 \mathrm{~km}$ al noroeste de Sechura (Fig. 1). El área aproximada es de 1000 ha, sin considerar la zona desértica y está dominado por dos especies de mangle, Laguncularia racemosa y Avicennia germinans

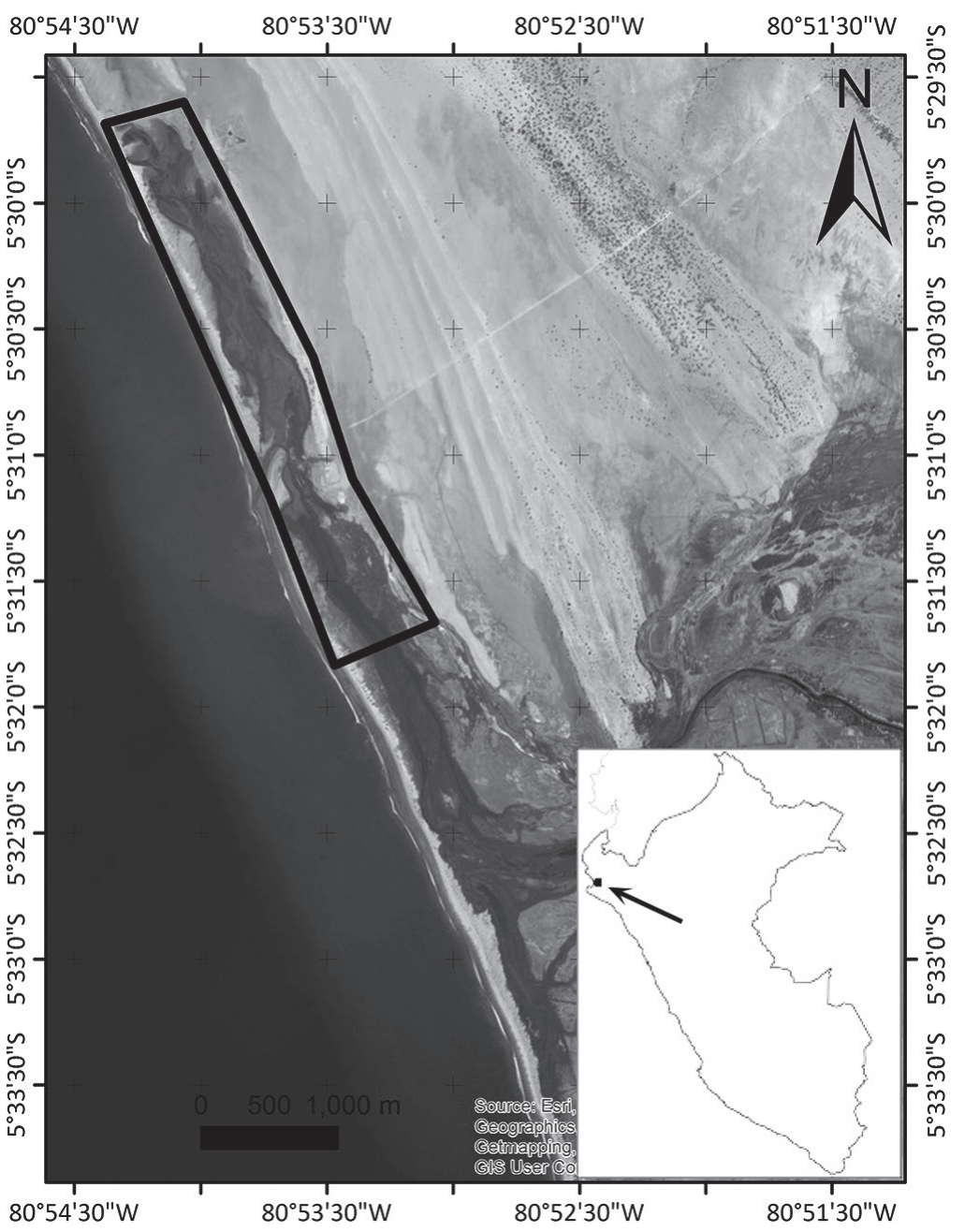

Figura 1. Ubicación del Manglar San Pedro de Vice. Piura - Perú. El polígono negro indica el área de muestreo. 
(Chávez-Villavicencio 2005). La precipitación media anual es de $30 \mathrm{~mm}$ con una temperatura media anual de $23^{\circ} \mathrm{C}$ (Hijmans et al. 2005).

Registro de datos.- Se realizaron 10 muestreos quincenales entre diciembre del 2010 y abril del 2011. En cada uno de ellos se recorrió una distancia de $4.5 \mathrm{~km}$ empezando desde el extremo norte del canal de marea, en el cual se contaron todos los individuos de aves limícolas. Los muestreos se ejecutaron preferentemente cuando la marea estaba bajando, para así minimizar el número de aves descansando en áreas próximas o adyacentes a la vegetación (Senner \& Angulo 2013). Para la identificación de las especies se emplearon binoculares (Nikon 10x42 y Vortex 10x42) y un telescopio (Kowa 60X) y para su determinación la guía de campo de Aves del Perú (Schulenberg 2010).

Análisis de datos.- Los promedios de las abundancias por especie, para establecer la dominancia durante el estudio, se compararon en un gráfico por sus intervalos de confianza al 95\%. La disminución de la riqueza de aves limícolas se analizó con una regresión de Poisson (enlace log) por tratarse de conteos, mientras que la disminución de la abundancia se hizo a través de los promedios de abundancias de cada fecha de muestreo con un modelo lineal generalizado (enlace identity). Los modelos lineales generalizados se elaboraron empleando el programa $\mathrm{R}$ v.3.4.0 (R Development Core Team 2017).

\section{Resultados}

Se registraron 19 especies de aves limícolas consideradas como migratorias neárticas (Tabla 1). Las especies Calidirs mauri, Calidris alba, Himantopus mexicanus y Pluvialis squatarola mostraron una tendencia a ser las más abundantes (en promedio), por lo tanto las especies dominantes del ensamble, aunque también fueron las que más variabilidad presentaron (Fig. 2).

Entre diciembre de 2010 y abril de 2011, tanto la riqueza de especies como la abundancia de aves limícolas migratorias neárticas mostraron una disminución estadísticamente significativa de acuerdo a los intervalos de confianza de las pendientes respectivas. En el caso de la riqueza de especies, la pendiente de

Tabla 1. Riqueza de especies y abundancia de aves limícolas registradas en el Manglar San Pedro de Vice entre diciembre de 2010 y abril de 2011

\begin{tabular}{|c|c|c|c|c|c|c|c|c|c|c|c|}
\hline $\mathbf{N}^{\mathrm{o}}$ & & 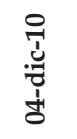 & 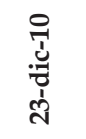 & 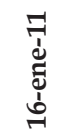 & 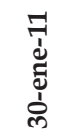 & 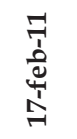 & 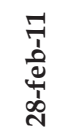 & 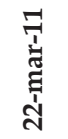 & 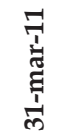 & 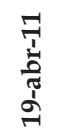 & 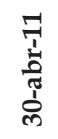 \\
\hline & Especie & \multicolumn{10}{|c|}{ Abundancias } \\
\hline I. & CHARADRIIDAE & & & & & & & & & & \\
\hline 1 & Pluvialis squatarola & 158 & 239 & 262 & 183 & 181 & 328 & 82 & 36 & 43 & 14 \\
\hline 2 & Charadrius semipalmatus & 3 & 30 & 16 & 14 & 10 & 28 & 2 & 20 & 12 & 16 \\
\hline 3 & Charadrius wilsonia & 11 & 32 & 23 & 11 & 6 & 7 & 9 & 18 & 0 & 0 \\
\hline 4 & Charadrius alexandrinus & 15 & 6 & 3 & 2 & 0 & 12 & 0 & 14 & 0 & 0 \\
\hline 5 & Charadrius collaris & 2 & 2 & 0 & 0 & 0 & 0 & 0 & 3 & 0 & 0 \\
\hline II. & HAEMATOPODIDAE & & & & & & & & & & \\
\hline 6 & Haematopus palliatus & 22 & 22 & 35 & 21 & 37 & 17 & 44 & 19 & 25 & 23 \\
\hline III. & RECURVIROSTRIDAE & & & & & & & & & & \\
\hline 7 & Himantopus mexicanus & 52 & 164 & 76 & 110 & 116 & 490 & 188 & 98 & 172 & 268 \\
\hline IV. & SCOLOPACIDAE & & & & & & & & & & \\
\hline 8 & Limnodromus griseus & 114 & 90 & 0 & 53 & 152 & 64 & 95 & 0 & 34 & 55 \\
\hline 9 & Limosa fedoa & 0 & 0 & 0 & 0 & 2 & 0 & 0 & 0 & 0 & 0 \\
\hline 10 & Numenius phaeopus & 26 & 44 & 31 & 15 & 27 & 22 & 62 & 25 & 19 & 21 \\
\hline 11 & Actitis macularius & 22 & 20 & 14 & 13 & 39 & 14 & 13 & 12 & 7 & 12 \\
\hline 12 & Tringa melanoleuca & 56 & 75 & 20 & 16 & 82 & 20 & 6 & 20 & 8 & 11 \\
\hline 13 & Tringa flavipes & 2 & 4 & 0 & 3 & 4 & 0 & 7 & 2 & 0 & 9 \\
\hline 14 & Tringa semipalmata & 88 & 191 & 115 & 132 & 180 & 139 & 8 & 10 & 12 & 25 \\
\hline 15 & Arenaria interpres & 89 & 45 & 77 & 20 & 108 & 40 & 74 & 66 & 55 & 38 \\
\hline 16 & Calidris alba & 118 & 673 & 168 & 136 & 116 & 395 & 16 & 128 & 11 & 3 \\
\hline 17 & Calidris pusilla & 0 & 85 & 70 & 15 & 19 & 20 & 0 & 3 & 0 & 0 \\
\hline 18 & Calidris mauri & 378 & 904 & 1065 & 617 & 348 & 626 & 0 & 10 & 0 & 7 \\
\hline \multirow[t]{4}{*}{19} & Calidris minutilla & 76 & 73 & 29 & 24 & 24 & 25 & 5 & 38 & 12 & 7 \\
\hline & Abundancia Acumulada & 1232 & 2699 & 2004 & 1385 & 1451 & 2247 & 611 & 522 & 410 & 509 \\
\hline & Promedio Abundancia & 65 & 142 & 105 & 73 & 76 & 118 & 32 & 27 & 22 & 27 \\
\hline & Total de especies por muestreo & 17 & 18 & 15 & 17 & 17 & 16 & 14 & 17 & 12 & 14 \\
\hline
\end{tabular}




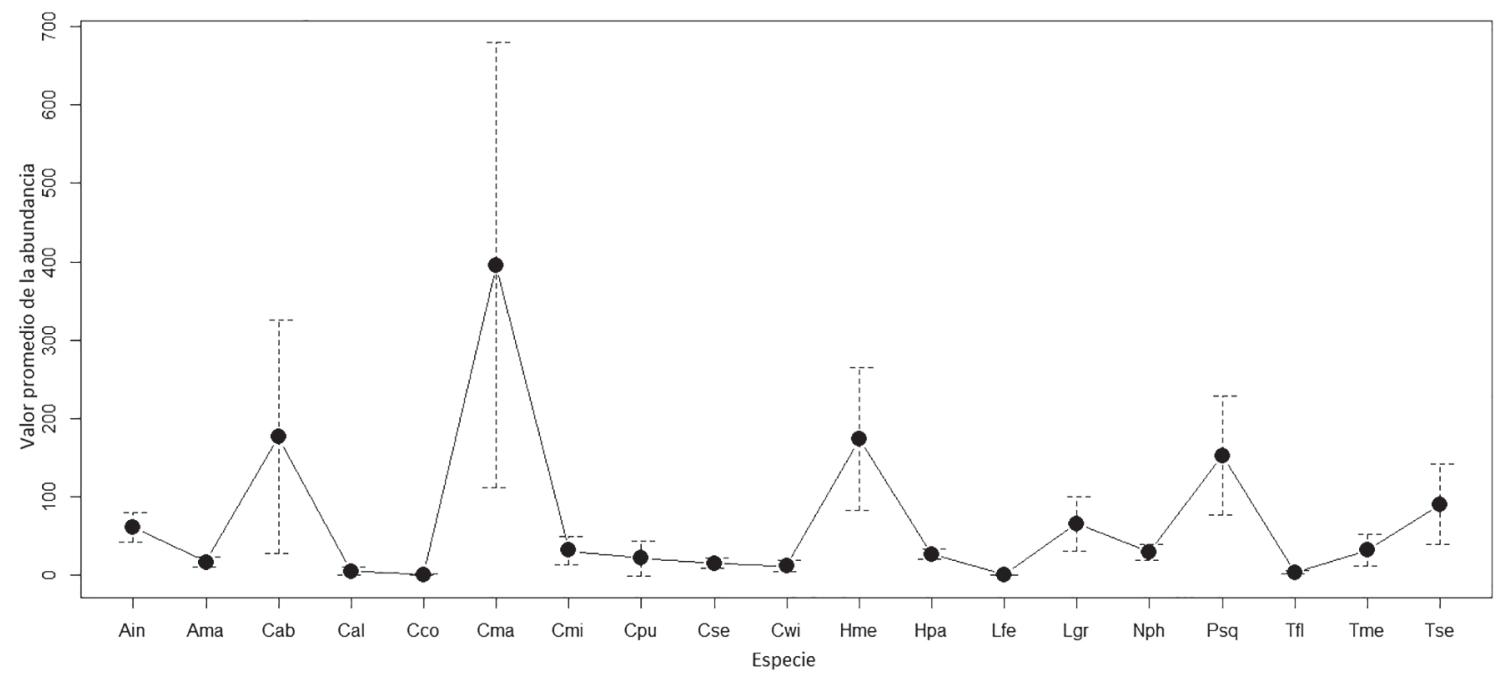

Figura 2. Variación del promedio de las abundancias de las especies limícolas migratorias neárticas del Manglar San Pedro de Vice (Piura - Perú). Diciembre 2010 - Abril 2011. Las barras corresponden al intervalo de confianza al 95\% (Códigos de especies en la Tabla 1).

la regresión de Poisson fue negativa $(b=-0.03)$, mientras que el intervalo de confianza al 95\% del valor interpretable no incluyó el cero (exp.b=0.97; IC95\%; 0.92 - 1.03). El modelo lineal generalizado aplicado a los promedios de las abundancias también presentó una pendiente negativa y su intervalo de confianza no incluyó el cero (b=-10.04; IC95\%; -16.85--3.22) (Figs. 3 y 4).

\section{Discusión}

La influencia positiva de las aves migratorias en la riqueza y abundancia de la riqueza aviar del Manglar San Pedro de Vice es evidente. Similar es la situación que ocurre en otros humedales costeros del hemisferio sur, en donde en los meses de verano y parte de otońo (diciembre - abril) hay un aumento en la diversidad de aves por la migración de esas especies (Pulido 1987, Hughes 1991, Keddy 2002, Chávez-Villavicencio 2005, Torres

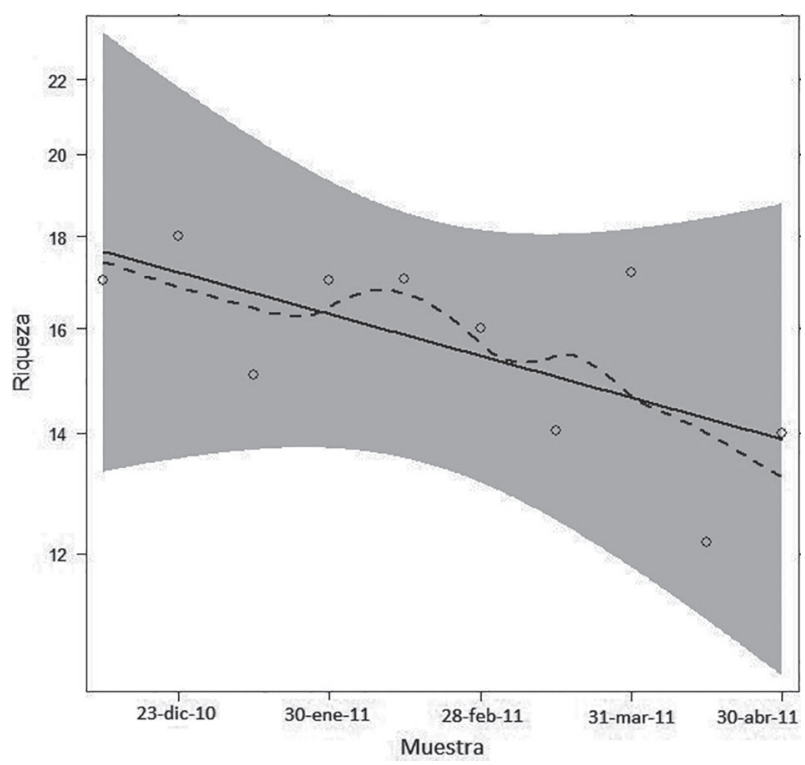

Figura 3. Relación negativa entre la riqueza de especies y el tiempo de muestreo. et al. 2006, Álvarez 2007, Vizcarra 2008, Gonzáles et al. 2011, Tabilo et al. 2015, Podestá et al. 2017).

Este trabajo registró 19 especies de aves limícolas migratorias neárticas entre los meses de verano y la mitad del otoño. GarcíaOlaechea y Chávez-Villavicencio (2014), reportaron para este mismo humedal un total de 29 especies de aves migratorias neárticas limícolas. Castro y Myers (1987) y Hughes (1991), señalaron que algunas especies de aves limícolas migratorias neárticas retrasan su partida e incluso no lleguen a migrar debido a que estos individuos son juveniles (no reproductivos) y que no acumulan grasa para su viaje de retorno. Estos factores, conjuntamente con el menor tiempo de muestro, podría explicar la diferencia encontrada entre el registro de aves de este estudio y el estudio de García-Olaechea y Chávez-Villavicencio (2014).

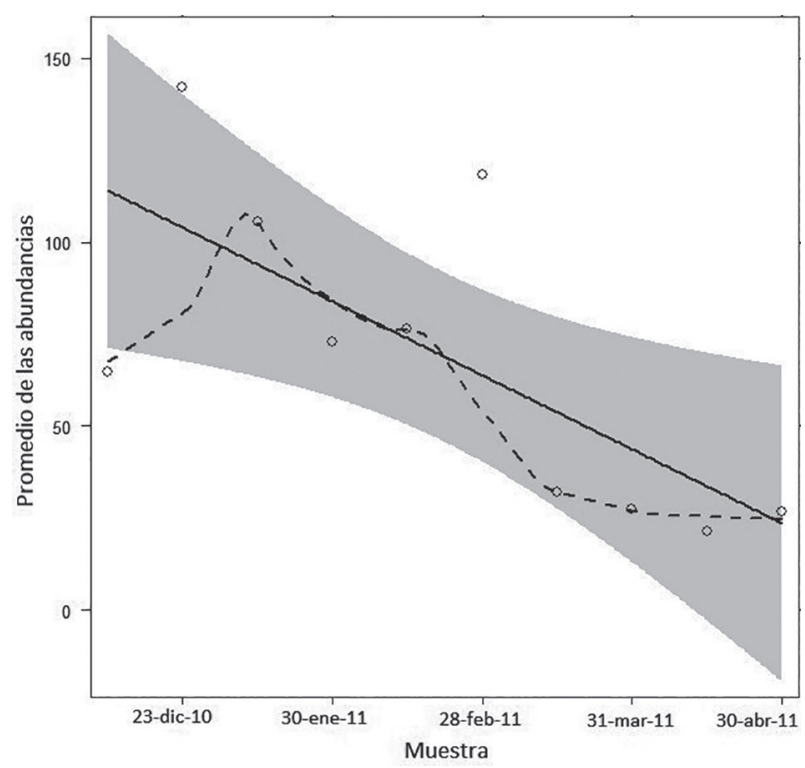

Figura 4. Relación negativa entre el promedio de las abundancias de especies y el tiempo de muestreo. 
La probabilidad de detección, los "singletons" y "doubletons" resultan importantes cuando se pretende establecer la influencia de un grupo de aves en los patrones estacionales de cualquier ecosistema, sobre todo cuando se involucran especies migratorias representadas por un individuo en un muestro, en este tipo de ecosistemas. Aunque Limosa fedoa para este estudio fue un "singleton", se comprobó que esta especie se registró en otras ocasiones en el manglar (eBird 2017).

En la Poza La Arenilla (Callao, Perú), un humedal artificial con agua de mar, Calidris himantopus, no generó ningún cambio en la abundancia de especies para el invierno y primavera en 2015 por haberse registrado solo un individuo (Podestá et al. 2017). Al respecto, se considera que pudo no haberse generado cambios en la abundancia general de las aves del humedal, pero necesariamente debió contribuir al incremento de la riqueza de especies. El tema central es si estas especies con registros únicos y con un solo individuo ("singletons"), deben ser considerados en los análisis del incremento o disminución de riqueza de especies en épocas de migración si solo se registraron una vez en la historia del humedal.

Los trabajos citados (Pulido 1987, Hughes 1991, Keddy 2002, Chávez-Villavicencio 2005, Torres et al. 2006, Álvarez 2007, Vizcarra 2008, Gonzáles et al. 2011, Tabilo et al. 2015, Podestá et al. 2017) atribuyeron la partida de las especies migratorias a la disminución de riqueza y abundancia de aves hacia el otońo e invierno, aunque incluyeron no solo a especies limícolas, sino también a especies de patos (Anatidae), gaviotas y gaviotines (Laridae). La variabilidad temporal de las especies migratorias y residentes en el Humedal de Ventanilla (Perú), también se atribuyó a que las especies migratorias fueron más abundantes entre los meses de noviembre y mayo, disminuyendo a partir de junio hasta julio (Álvarez 2007). Aunque no se detalla sobre especies limícolas, de su listado se desprende la presencia de 16 especies pertenecientes a este grupo de aves que habrían influido, al migrar, a la disminución de la riqueza de aves del humedal.

Un trabajo en los Pantanos de Villa (Lima - Perú) referido específicamente a aves limícolas (Torres et al. 2006), reportó que en septiembre se observó un aumento de la abundancia promedio debido principalmente a la llegada de especies migratorias, que permanecieron en el humedal hasta su próxima migración. Ese trabajo registra un segundo aumento de abundancia promedio en enero, mayor que el registrado en septiembre y lo atribuyó al nacimiento de las primeras crías Himantopus mexicanus y Charadrius vociferus, las cuales iniciaron su periodo reproductivo a finales de octubre, mientras que las migratorias Calidris pusilla, Tringa flavipes y Charadrius semipalmatus, incrementaron el número de individuos en este mes. Resultados similares encontraron Podestá et al. (2017) en la Poza La Arenilla, ubicada 23 $\mathrm{km}$ al noroeste de los Pantanos de Villa.

Ante la evidencia bibliográfica y los resultados de este estudio, se concluye que las aves limícolas migratorias neárticas sí influyen en los patrones estacionales de los ensambles de aves en los humedales costeros en Perú, incrementando su riqueza y abundancia cuando inician sus llegadas y disminuyéndolas cuando parten hacia el hemisferio norte a reproducirse.

\section{Agradecimientos}

Al Servicio Forestal de los Estados Unidos (USFS) y a CRIMBI (Copper River International Migratory Bird Initiative) por financiar este estudio. A los ex alcaldes Armando Bancayán Amaya, Agustín Eche Temoche y al personal de serenazgo de la Municipalidad Distrital de Vice por el apoyo en traslados al manglar, seguridad y disponibilidad ante cualquier emergencia. A Luiggi Quevedo, Jorge Novoa, Danny Silva, Antonio García, Kárlom Herrera y Elio Núñez por su compañía y esfuerzo al momento de realizar los conteos.

\section{Literatura citada}

Álvarez C. 2007. Evaluación de la diversidad especifica de las aves de los humedales de Ventanilla, Callao, Perú. Tesis para optar el título de licenciado en Ciencias Biológicas. Universidad Ricardo Palma. Lima, Perú.

Angulo F. 2009. IBAs de Perú. Pp 307 - 316. In: Devenish, C., Diaz Fernandez, D. F., Clay, R. P., Davidson, I. yYepez Zabala, I. Eds. Important Bird Areas Americas - Priority sites for biodiversity conservation. Quito, Ecuador: BirdLife International (BirdLife Conservation Series No 16)

Bildstein K.L., G.T. Bancroft, D.H. Dugan, R.M. Gordon, R.M. Edwin, E. Noel, L.X. Payne \& S.E. Senner. 1991. Approaches to the conservation of coastal wetlands in the Western Hemisphere. Wilson Bulletin 103(2): 218-254.

Canevari P., G. Castro, M. Sallabery \& L. Naranjo. 2001. Guía de los Chorlos y Playeros de la Región Neotropical. American Bird Conservancy, WWF- US, Humedales para las Américas y Manomet Conservation Science, Asociación Calidris. Santiago de Cali, Colombia.

Castro G. \& J. Myers. 1987. Ecología y conservación del playero blanco (Calidris alba) en el Perú. Boletín de Lima. (52): 47- 60.

Chávez-Villavicencio C. 2005. Variación estacional y uso de hábitat de las aves de los manglares de San Pedro (Sechura - Perú). Enero - diciembre 2004. Tesis para optar el título de Biólogo. Facultad de Ciencias, Universidad Nacional de Piura, Perú.

eBird. 2017. (En Línea) eBird: Una base de datos en línea para la abundancia y distribución de las aves [aplicación de internet]. eBird, Ithaca, New York. <http://www.ebird.org>. Acceso 19/4/2017

Gauthier G., J.F. Oisgiroux, A. Reed, A. Be'chet \& L. Langer. 2005. Interactions between land use, habitat use, and population increase in greater snow geese: what are the consequences for natural wetlands? Global Change Biology 11(6):856-868. doi: $10.1111 /$ j. $1365-2486.2005 .00944 . x$

García-Olaechea A. \& C. Chávez-Villavicencio. 2014. Nuevos registros de aves en el Sitio Ramsar Manglares de San Pedro de Vice, Piura - Perú. Boletín UNOP 9 (3): 16-24.

Gonzáles A., M. Vukasovic, \& C. Estades. 2011. Variación temporal en la abundancia y diversidad de aves en el humedal del Río Itata, región del Bío-Bío, Chile. Gayana 75(2): 170-181. http://dx.doi.org/10.4067/S0717-65382011000200006

Hijmans R. J., S. E. Cameron, J. L. Parra, P. G. Jones \& A. Jarvis. 2005. Very high resolution interpolated climate surfaces for global land areas. International Journal of Climatology 25:1965-1978. https://doi.org/10.1002/joc. 1276

Hughes R.A. 1991. Las aves de la provincia de Islay. Boletín de Lima 75: 47-54.

Keddy P.A. 2002. Wetland Ecology: Principles and Conservation. Cambridge University Press, Great Britain.

Koepcke M. 1964. Las aves del departamento de Lima. Talleres Gráfica Morson S.A. Lima - Perú. 118 pp.

Kushlan J.A. 1993. Waterbirds as bioindicators of wetland change: are they a valuable tool?. Pp. 48-55 In: Moser, M., R.C. Prentice \& J. van Vessem (eds.). Waterfowl and Wetland Conservation in the 1990s - A global perspective. IWRB Spec. Publ. No. 26. Slimbridge, UK.

Podestá J., A. Cotillo, E. Segura-Cobeña \& G. Cabanillas. 2017. Variación temporal de la riqueza y abundancia de aves playeras limícolas en el humedal costero "Poza de la Arenilla"- La Punta, Callao. The Biologist (Lima), 15: 23-35.

Pulido V. 1987. Patrones de variación estacional de las poblaciones de aves de las lagunas de Mejia. UNALM. Escuela de Postgrado. Especialidad de conservación de recursos forestales. Tesis para optar el grado de Magíster Scientiae. Universidad Agraria Nacional La Molina. Lima, Perú. 
Pulido V. 2003. Influencia de la pérdida de hábitats en la conservación de las aves de los Pantanos de Villa. Tesis para optar el grado académico de Doctor en Ciencias Biológicas. Universidad Nacional Mayor de San Marcos. Lima, Perú.

R Core Team. 2017. R: A language and environment for statistical computing. R Foundation for Statistical Computing, Vienna, Austria. <URL https://www.R-project.org/>. Aceso $15 / 4 / 2017$

The Ramsar Convention on Wetlands. 2016. RAMSAR Sites Information Service <http://ramsar. wetlands.org/Database/ Searchforsites/tabid/765/language/en-US/Default.aspx $>$. Acceso 28/07/2016.

Rappole J., E. Morton, T. Lovejoy \& J. Ruos. 1993. Aves migratorias neárticas en los neotrópicos. 1ra. Ed. RR Donnelley y Sons Co. Washington - USA

Red Hemisférica de Reservas de Aves Playeras - RHRAP. 2012. Lista de sitios. <http://www.whsrn.org/es/ perfil-de-sitio/manglaresde-san-pedro-de-vice>. Acceso 28/07/2012.
Senner N.R. \& F. Angulo. 2013. Atlas de las aves playeras del Perú. Sitios importantes para su conservación. CORBIDI. Lima, Perú.

Schulenberg T.S., D.F. Stotz, D.F. Lane, J.P. O’Neill, and T.A. Parker III. 2010. Aves de Perú. Serie Biodiversidad Corbidi 01. CORBIDI, Lima, Perú, pp.1-660

Tabilo E. J. Burmeister, C. Chávez-Villavicencio \& C. Zöckler. 2016. Humedales y aves migratorias en la costa árida del Pacífico sudamericano. Etapa 1. Centro Neotropical de Entrenamiento en Humedales y Manfred Hermsen Stiftung. Coquimbo - Chile.

Torres M. Z. Quinteros \& F. Takano. 2006. Variación temporal de la abundancia y diversidad de aves limícolas en el refugio de vida silvestre Pantanos de Villa, Lima - Perú. Ecología Aplicada 5: 119-125.

Vizcarra J. 2008. Composición y conservación de las aves en los humedales de Ite, suroeste del Perú. Boletín Chileno de Ornitología 14(2): 59-80. 